\title{
OPEN Untangling the genetic link between type 1 and type 2 diabetes using functional genomics
}

\author{
Denis M. Nyaga ${ }^{1}$, Mark H. Vickers ${ }^{1}$, Craig Jefferies ${ }^{1,2}$, Tayaza Fadason ${ }^{1}$ \& Justin M. O'Sullivan ${ }^{1,3 凶}$
}

There is evidence pointing towards shared etiological features between type 1 diabetes (T1D) and type 2 diabetes (T2D) despite both phenotypes being considered genetically distinct. However, the existence of shared genetic features for T1D and T2D remains complex and poorly defined. To better understand the link between T1D and T2D, we employed an integrated functional genomics approach involving extensive chromatin interaction data $(\mathrm{Hi}-\mathrm{C})$ and expression quantitative trait loci (eQTL) data to characterize the tissue-specific impacts of single nucleotide polymorphisms associated with T1D and T2D. We identified 195 pleiotropic genes that are modulated by tissue-specific spatial eQTLs associated with both T1D and T2D. The pleiotropic genes are enriched in inflammatory and metabolic pathways that include mitogen-activated protein kinase activity, pertussis toxin signaling, and the Parkinson's disease pathway. We identified 8 regulatory elements within the TCF7L2 locus that modulate transcript levels of genes involved in immune regulation as well as genes important in the etiology of T2D. Despite the observed gene and pathway overlaps, there was no significant genetic correlation between variant effects on T1D and T2D risk using European ancestral summary data. Collectively, our findings support the hypothesis that T1D and T2D specific genetic variants act through genetic regulatory mechanisms to alter the regulation of common genes, and genes that co-locate in biological pathways, to mediate pleiotropic effects on disease development. Crucially, a high risk genetic profile for T1D alters biological pathways that increase the risk of developing both T1D and T2D. The same is not true for genetic profiles that increase the risk of developing T2D. The conversion of information on genetic susceptibility to the protein pathways that are altered provides an important resource for repurposing or designing novel therapies for the management of diabetes.

Type 1 diabetes (T1D) and type 2 diabetes (T2D) are both complex polygenic metabolic disorders, which are generally considered to be pathophysiologically and genetically distinct entities. However, there is some evidence pointing towards T1D and T2D sharing common etiological features (e.g. apoptosis of pancreatic islet beta cells) resulting in insulin deficiency ${ }^{1-3}$. In young adults, the increase in obesity rates is making it difficult to differentiate between T1D and T2D ${ }^{4}$. Moreover, the latent autoimmune diabetes in adults (LADA) phenotype appears to be an intermediate phenotype that refers to individuals who initially have clinical features that are similar to T2D, but develop autoimmunity towards islet cells leading to progressive beta-cell failure late in life ${ }^{5,6}$. Collectively, these observations may support an overlap in the pathogenesis of both T1D and T2D, but whether this is due to environmental, genetic, or biological pathway intersections, or a combination of these effects remains to be determined.

Comprehensive genome-wide association studies (GWAS) have uncovered distinct and shared loci, marked by single nucleotide polymorphisms (SNPs), which are associated with the development of T1D and T2D ${ }^{7-10}$. Interestingly, Li et al. ${ }^{11}$ suggested that this possible genetic interplay between T1D and T2D could be mediated by the human leukocyte antigen (HLA) region. The HLA locus, which accounts for $\sim 50 \%$ of the genetic risk for $\mathrm{T}_{1} \mathrm{D}^{12}$, has been associated with both T2D susceptibility ${ }^{13,14}$ and T2D protection ${ }^{15}$. Furthermore, genetic variants within the transcription factor 7-like 2 (TCF7L2) have been strongly associated with T2D and LADA ${ }^{16,17}$, yet the clinical presentation of LADA is similar to T1D (i.e. autoantibody positivity in LADA patients) ${ }^{17,18}$.

The hypothesized existence of shared genetic features in individuals with T1D and T2D indicates that loci act to predispose or protect individuals to one or both of the phenotypes of diabetes-either cumulatively or inversely ${ }^{19,20}$. Such a scenario is not unexpected if one considers the regulation of the insulin secretion, signaling

${ }^{1}$ Liggins Institute, The University of Auckland, Auckland, New Zealand. ${ }^{2}$ Starship Children's Health, Auckland, New Zealand. ${ }^{3}$ The Maurice Wilkins Centre, The University of Auckland, Auckland, New Zealand. ${ }^{\square}$ email: justin.osullivan@auckland.ac.nz 
and response pathways as a whole and not as separate modules. Thus, even though the risk alleles for both T1D and T2D may be different, the impacts on gene regulation and biological pathways may converge in both phenotypes: as ultimately diabetes is caused by a lack of insulin action (a relative or absolute deficiency ${ }^{21}$ ).

We have previously reported that SNPs associated with T2D mark regulatory loci that physically interact with-and act as expression quantitative trait loci (eQTLs) for-genes involved in the leptin and insulin signaling pathways ${ }^{22}$. Furthermore, we subsequently demonstrated that SNPs associated with T1D spatially regulate the expression of genes involved in immune system activation and responses ${ }^{23}$. As such, a greater understanding of how differences in gene regulation contribute to the observed etiological and pathophysiological similarities between T1D and T2D would aid in the management and treatment of diabetes. Here, we characterize the biological pathway overlaps for genes regulated by unique and shared SNPs associated with genetic risk for the development of T1D and T2D.

\section{Results}

T1D and T2D-associated SNPs form an overlapping gene regulatory network. Previously, we reported that SNPs associated with the development of T1D and T2D mark gene regulatory elements that modulate gene transcript levels ${ }^{22,23}$. Here, we sought to investigate if T1D and T2D share tissue-specific regulatory networks. Emerging evidence indicates that complex diseases culminate from systems-level perturbations $\mathrm{s}^{24,25}$. Therefore, using the CoDeS3D algorithm ${ }^{26}$ (Methods), we integrated extensive chromatin interaction (Hi-C; Supplementary Table S1) and eQTL data across multiple human tissues. We identified 1,796 and 2,831 unique pairs of spatial eQTLs (T1D: Supplementary Table S2; T2D: Supplementary Table S3; Supplementary Fig. S1) involving 346 and 1,569 T1D and T2D GWAS SNPs at FDR <0.05, respectively (Supplementary Table S4; Supplementary Table S5). Consistent with our earlier observations ${ }^{22,23}$, ontological analyses (using the $\mathrm{R}$ software package; g:Profiler ${ }^{27}$ [Methods]) of the genes that were impacted by the spatial eQTLs identified significant enrichment in immune system response and metabolic signaling pathways (FDR $<0.05$ ), for T1D and T2D, respectively (Supplementary Table S6; Supplementary Table S7).

It is possible that there is heterogeneity in the groups of SNPs obtained from the GWAS Catalog such that particular SNPs are associated with complications for all forms of diabetes (e.g. LADA and fulminant T1D). The inclusion of SNPs associated with complications in each of the T1D and T2D SNP sets may cause spurious results due to the existence of the identical SNP. Therefore, we tested for the presence of SNPs that were repeated in the T1D and T2D SNP sets we obtained from the GWAS catalogue. We identified 12 identical GWAS SNPs (i.e. $3.5 \%$ and $0.8 \%$ of T1D and T2D SNPs, respectively) that were present in both datasets (Fig. 1a). Bootstrapping indicated that this overlap does not occur by chance (Fig. 1b), consistent with the idea of shared complications. Notably, 7 of the 12 identical GWAS SNPs were spatial eQTLs (Supplementary Fig. S2).

Colocalization analyses test if two signals (i.e. disease vs cis-eQTL or trait vs trait) share the same 'causal' gene or $\mathrm{SNP}^{28}$. Bayesian colocalization tests between spatial cis-eQTL signals and disease-associated signals were conducted for genomic regions marked by the 7 identical SNPs that were strongly associated $\left(p<10^{-8}\right)$ with both T1D and T2D (i.e. SH2B3, MAPK14, CTRB1/2, INS, ASCL2/MIR4686, and HLA region). There was no evidence of complete colocalization between the disease and eQTL signals as defined by PP3 + PP4 $\geq 0.99$ and PP4/ PP3 $\geq 5$, a cut off previously suggested ${ }^{29}$ (Methods; Supplementary Table S8). However, we found weak evidence for colocalization between eQTL and GWAS signals for CTRB1/2 loci (rs7202877; posterior probability $=35.2 \%$ ) in both diseases, and SH2B3 loci (rs3184504; posterior probability=34.7\%) in T1D (Supplementary Table S8). Only 3 regions associated with both traits (i.e. CTRB1/2, SH2B3, and HLA loci) were found to share a causal SNP (Supplementary Table S8), indicating that T1D and T2D are driven by independent genetic signals. Collectively, this is consistent with the fact that 5 are associated with diabetic foot ulcers, 4 are associated with latent autoimmune diabetes (as well as associated with primary T1D), and 3 associated with primary T1D or T2D).

We reasoned that T1D and T2D would share features that are due to regulatory effects on common pleiotropic genes by SNPs specific to each condition. We identified a total of 195 shared genes $(20 \%$ and $12 \%$ of T1D and T2D genes, respectively) that were modulated by spatial eQTLs associated with T1D and T2D (Fig. 1c; Supplementary Fig. S2). Only 48 shared genes resulted from the 7 eQTLs that were due to identical SNPs. Bootstrapping confirmed that the observed overlap of 195 genes was non-random (Fig. 1d), consistent with the hypothesis that the regulatory effects are on genes that have pleiotropic effects on T1D and T2D.

We examined the 195 shared genes to identify which biological pathways and processes they are involved in. Notably, the subset of 165 shared genes, which excluded 30 classical and non-classical HLA genes, were enriched for pathways that include mitogen-activated protein kinase (MAPK), pertussis and Parkinson's disease pathways (Table 1). MAPK activity is important in the regulation of pancreatic beta cell function and insulin signaling ${ }^{30-32}$, and beta cell death through inflammatory responses in islet cells ${ }^{33}$. Additionally, pertussis toxin has been implicated in the regulation of insulin secretion from pancreatic beta cells through heterotrimeric $\mathrm{G}$ proteins ${ }^{34-36}$. Finally, $a$-Synuclein, a protein central to Parkinson's diseas ${ }^{37,38}$, has been shown to regulate insulin secretion in beta cells ${ }^{39}$. Collectively, our results support the hypothesis that spatial gene regulatory networks contribute to shared genetic risk between T1D and T2D.

SNPs used in polygenic risk scores for T1D and T2D modulate transcript levels of genes with pleiotropic effects. It remains possible that the inclusion of GWAS for, and SNPs associated with, diabetic complications in both the T1D and T2D SNP sets drives the common features we observed within the spatialeQTLs (Section "T1D and T2D-associated SNPs form an overlapping gene regulatory network"). Therefore, we sought to understand whether highly predictive SNPs used in polygenic risk scores for T1D and T2D $\mathrm{D}^{40,41}$ are involved in transcriptional co-regulation of genes associated with both diseases. In a polygenic risk score analysis for T1D, Sharp et al. included 67 imputed and genotyped T1D SNPs to predict early-onset T1D with 96\% 
a)
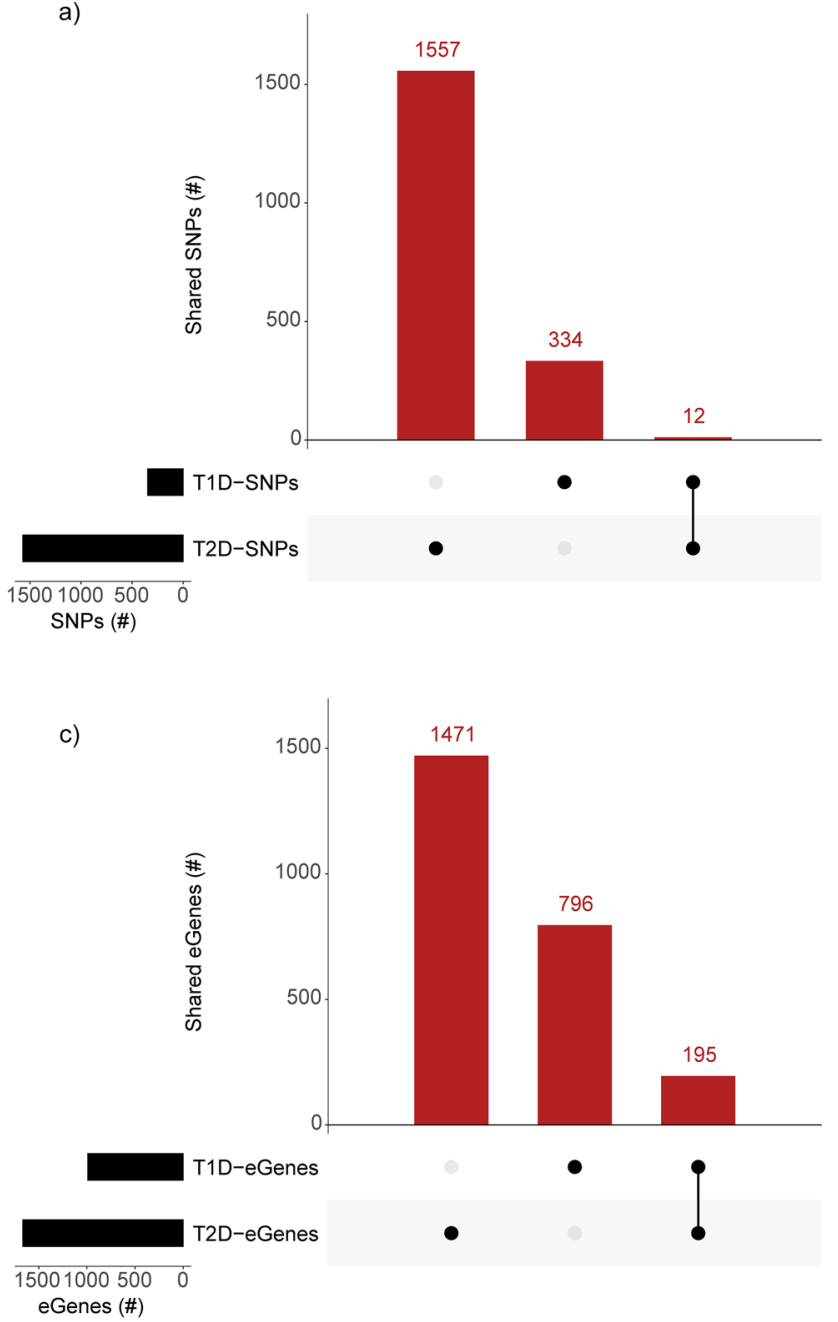

b)

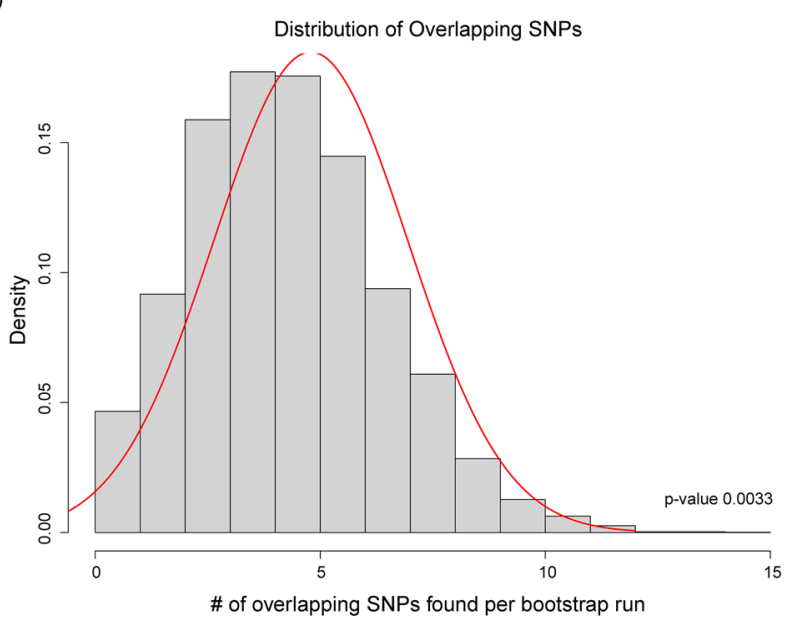

d)

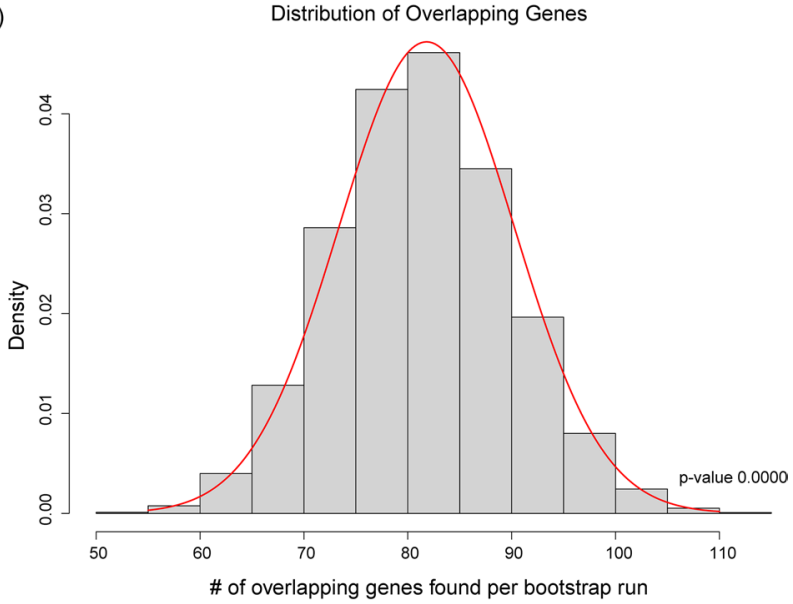

Figure 1. The co-regulation of pleiotropic genes associated with T1D and T2D is non-random. (a) A plot showing the number of unique and shared SNPs (imputed and genotyped) associated with the development of T1D and T2D identified from genome-wide association studies. (b) A normal distribution plot for randomly sampled SNPs (bootstrapping; $\mathrm{n}=10,000$ ) validates the significance of overlaps seen in (a). (c) The numbers of unique and shared genes modulated by T1D and T2D regulatory SNPs. (d) A normal distribution plot for randomly sampled genes (bootstrapping; $\mathrm{n}=10,000$ ) validates the significance of overlaps seen in (c). eGene-a gene whose transcript levels is associated with an eQTL, eQTL-SNPs associated with transcript levels of genes. Red lines in (b) and (d) illustrates the fitted normal distribution lines.

\begin{tabular}{|l|l|l|l|l|l|l|}
\hline id & Source & Term id & Term name & Term size & Intersection size & Corrected $\boldsymbol{p}$ values \\
\hline 1 & CORUM & CORUM:6307 & RAB27A-SLP3-KLC1 transport complex & 3 & 2 & 0.049 \\
\hline 2 & GO:MF & GO:0005524 & ATP binding & 1499 & 24 & 0.023 \\
\hline 3 & GO:MF & GO:0035639 & Purine ribonucleoside triphosphate binding & 1845 & 27 & 0.033 \\
\hline 4 & GO:MF & GO:0032559 & Adenyl ribonucleotide binding & 1557 & 24 & 0.042 \\
\hline 5 & GO:MF & GO:0030554 & Adenyl nucleotide binding & 1568 & 24 & 0.046 \\
\hline 6 & GO:MF & GO:0004707 & MAP kinase activity & 14 & 3 & 0.049 \\
\hline 7 & KEGG & KEGG:05133 & Pertussis & 76 & 5 & 0.016 \\
\hline 8 & WP & WP:WP2371 & Parkinson's Disease Pathway & 38 & 4 & 0.032 \\
\hline
\end{tabular}

Table 1. Significant biological and functional enrichment for pleiotropic genes associated with T1D and T2D. Corum, comprehensive resource of mammalian protein complexes; MF, molecular function; KEGG, Kyoto Encyclopedia of Genes and Genomes; WP, WikiPathways. 
a)
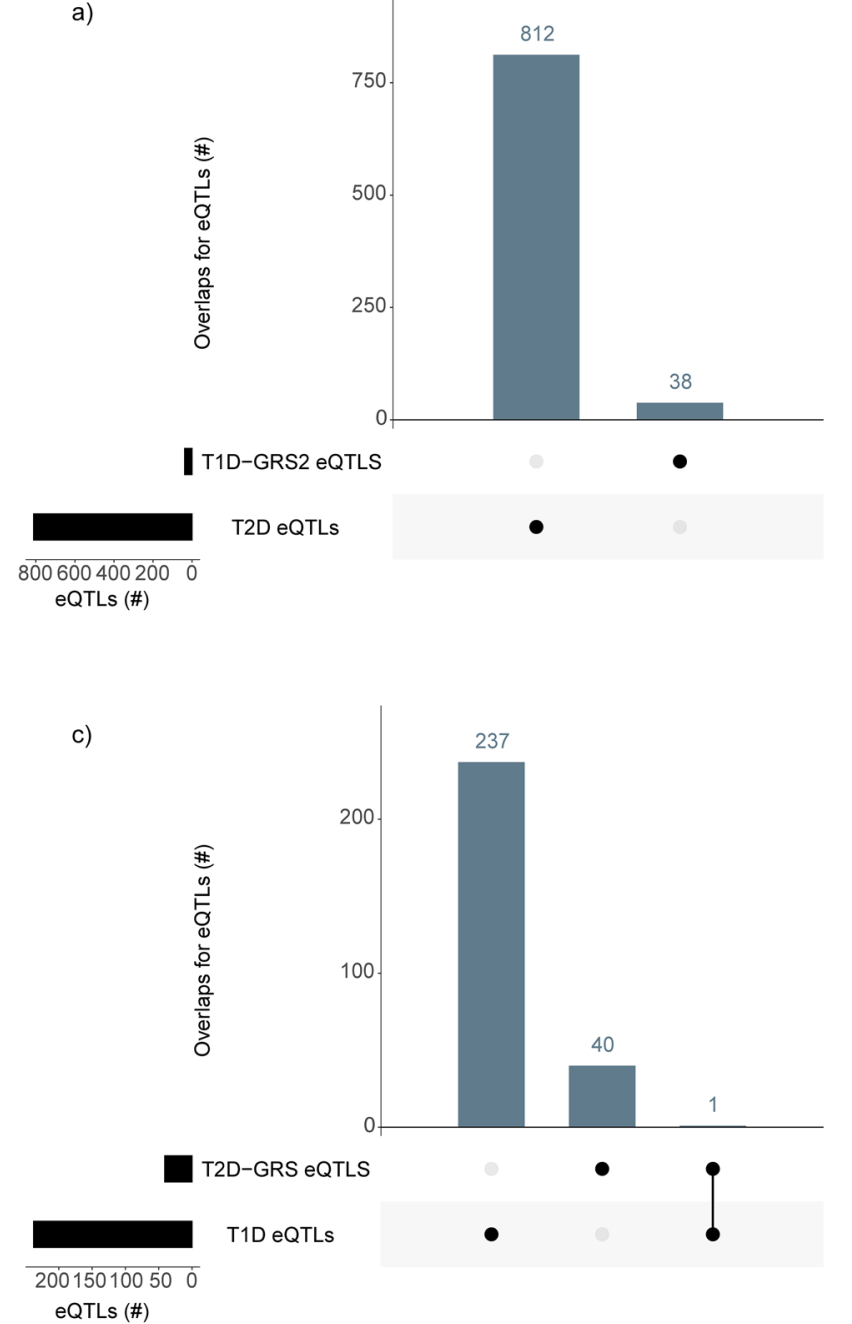
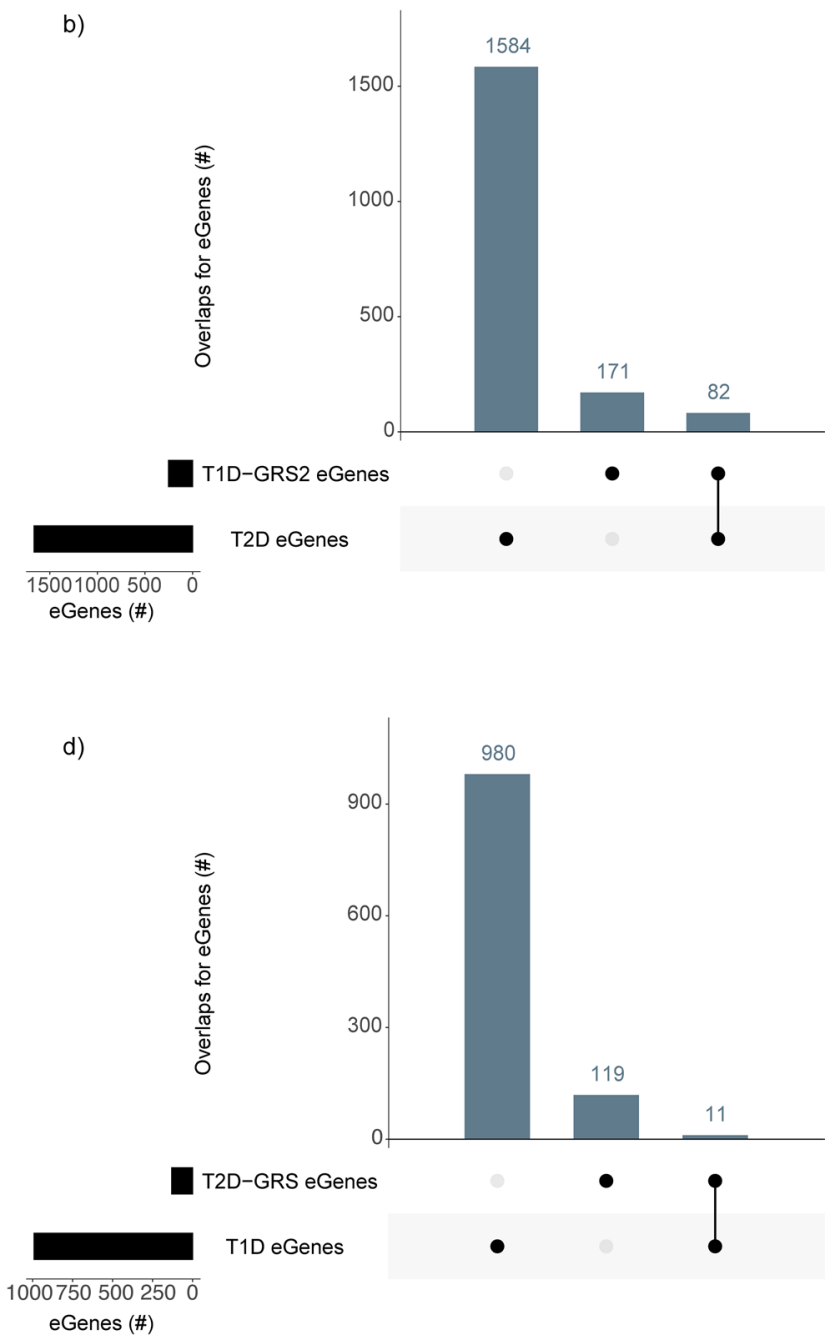

Figure 2. Highly predictive SNPs in polygenic risk scores for T1D and T2D are involved in transcriptional co-regulation of genes that mediate pleiotropic effects. (a) The number of unique and shared eQTLs for T1DGRS2 and T2D SNPs. (b) The number of unique and shared genes regulated by spatial regulatory elements marked by T1D-GRS2 and T2D eQTLs. (c) The number of unique and shared eQTLs for T2D-GRS and T1D SNPs. (d) The number unique and shared genes regulated by regulatory elements marked by T2D-GRS and T1D eQTLs. T1D-GRS2-type 1 diabetes genetic risk score 2, T2D-GRS-type 2 diabetes genetic risk score.

accuracy (i.e. T1D genetic risk score 2 [T1D-GRS2]) ${ }^{40}$. There were no identical SNPs between the TID-GRS2 and the T2D-associated SNPs lists (Supplementary Table S9). From our CoDeS3D analysis, we found that 38 of the 67 SNPs from T1D-GRS2 are spatial eQTLs that mark regulatory regions for 253 genes (Fig. 2a, b; Supplementary Table S10). Notably, despite no overlap between the T1D-GRS2 and T2D spatial eQTLs (Fig. 2a), we identified 82 shared genes (excluding HLA genes) that were associated with both T1D and T2D (Fig. 2b).

In a polygenic risk score analysis for T2D (T2D-GRS), 62 T2D-associated SNPs were combined with age, sex, and clinical risk factors to predict T2D development with $91 \%$ accuracy ${ }^{41}$. Two SNPs were identical between the T2D-GRS and the T1D-associated SNP lists (Supplementary Table S9). From our CoDeS3D analysis, we identified that 41 of the 62 SNPs from the T2D-GRS mark spatial regulatory elements for 130 genes (Fig. 2c,d; Supplementary Table S11). Notably, we identified 11 shared genes between T2D-GRS and the T1D-associated eQTLs (Fig. 2d), of which 9 were associated with the identical eQTL (rs7202877; Fig. 2c).

It is notable that the comparison of genes associated with T1D-GRS2 vs T2D-associated eQTLs showed greater overlap than the comparison of genes associated with T2D-GRS vs T1D-associated eQTLs (Fig. 2b, d, respectively). Collectively, our findings are consistent with the hypothesis that the highly predictive SNPs used in polygenic risk scores for T1D are involved in transcriptional co-regulation of genes that mediate pleiotropic effects in both T1D and T2D.r

T1D and T2D variant heritability is not significantly correlated in Europeans. We observed that GWAS SNPs associated with T1D and T2D are involved in transcriptional co-regulation of pleiotropic genes from the CoDeS3D analysis. Therefore, we employed the high-definition likelihood (HDL) method ${ }^{42}$ to calculate the genetic correlation between variant effects on T1D and T2D risk using European ancestral summary data 


\begin{tabular}{|l|l|l|l|}
\hline Phenotype 1 & Phenotype 2 & $\boldsymbol{r}_{\boldsymbol{g}}($ s.e. $)$ & $\boldsymbol{p}$ value \\
\hline Type 1 diabetes & Type 2 diabetes & $0.17(0.09)$ & $5.9 \times 10^{-2}$ \\
\hline Type 1 diabetes & BMI & $0.04(0.03)$ & $2.2 \times 10^{-1}$ \\
\hline Type 1 diabetes & Obesity & $0.08(0.09)$ & $4.4 \times 10^{-1}$ \\
\hline Type 2 diabetes & BMI & $0.48(0.04)$ & $5.5 \times 10^{-40 *}$ \\
\hline Type 2 diabetes & Obesity & $0.31(0.09)$ & $7.9 \times 10^{-4 \star}$ \\
\hline Obesity & BMI & $0.65(0.06)$ & $2.9 \times 10^{-25 *}$ \\
\hline
\end{tabular}

Table 2. Genetic correlation estimates between T1D, T2D, body mass index and obesity in people of European ancestry. $r_{g}$, genetic correlation estimate; s.e., standard error; $p$ value, Bonferroni corrected $p$ values; ${ }^{*}$ significant corrected $p$ values. Variant heritability estimates (h2; s.e.) are: T1D (0.0046; 9e-04), T2D (0.01; 9e-04), BMI (0.2565; 0.0081), Obesity (0.0061; 0.0011).

from the UK Biobank (UKBB). The HDL method robustly increases the precise estimation of genetic correlation between phenotypes, and estimates variant heritability, through its extensive inclusion of genome-wide linkage disequilibrium ${ }^{42}$. HDL analysis did not identify a genetic correlation between T1D and T2D $\left(r_{g}=0.17 ; p\right.$ value $\left.=5.9 \times 10^{-2}\right)($ Table 1$)$. T1D did not correlate with either body mass index, or obesity (Table 2$)$. However, consistent with previous observations by Carlsson et al. ${ }^{43}$, we observed a significant positive correlation between T2D and body mass index, and between T2D and obesity (Table 2). These results are consistent with the interpretation that the overlap of biological mechanisms between T1D and T2D occurs at the level of gene control and not at the level of variant heritability.

The TCF7L2 locus is a spatial regulatory hub for genes important for immune regulation and T2D etiology. TCF7L2 is a knownT2D susceptibility locus ${ }^{44}$ that encodes a transcription factor that is central to the Wnt signaling pathway. SNPs mapped within TCF7L2 have also been associated with the presence of islet autoantibodies in $\mathrm{LADA}^{6,16-18}$ and recent-onset T1D patients ${ }^{45}$. As such, TCF7L2 has been hypothesized to be the key to understanding the genetic link between the pathogenesis of T1D and T2D ${ }^{20}$. We hypothesized that TCF7L2 is a spatial regulatory hub for genes important for the etiology of T1D and T2D. Our CoDeS3D analysis identified 8 regulatory elements marked by SNPs within the TCF7L2 locus (Supplementary Table S12). Four of these eQTLs (i.e. rs34872471, rs7901695, rs4506565, rs7903146) coordinate the regulation of TCF7L2 expression (Fig. 3a; Supplementary Table S12). Notably, rs4506565 is associated with single autoantibody in recent-onset $\mathrm{T}_{1} \mathrm{D}^{45}$. The 4 regulatory SNPs are also in strong linkage $\left(\mathrm{R}^{2}>0.8\right)$ across the European population. Rs7903146 overlaps histone modification marks and an annotated enhancer in the pancreas (Fig. 3b).

Notably, our CoDeS3D analysis identified regulatory elements within the TCF7L2 locus that were associated with trans-regulation of genes involved in the regulation of immune responses, including PLCG2, ZEB1, and ROBO1 (Fig. 3; Supplementary Table S12). PLCG2 encodes a phospholipase implicated in inflammation and autoimmunity ${ }^{46}$, and in $\mathrm{T}$ cell function and selection ${ }^{47}$. $R O B O 1$ expression has been hypothesized to serve as a biomarker for T1D diagnosis due to its regulatory role in the recruitment of diabetogenic $\mathrm{T}$ cells ${ }^{48}$. Additionally, ZEB1, which is also spatially regulated in cis by a T1D-eQTL (i.e. rs2793108-81 Mb away from the TCF7L2 locus; Supplementary Table S2), encodes a zinc finger transcription factor that functions as a key regulator of the $\mathrm{T}$ cell signaling and differentiation in the thymus ${ }^{49}$.

Interestingly, we also identified a SNP within TCF7L2 (i.e. rs17746147) that modulated the expression of genes involved in insulin signaling (i.e. SCD) (Supplementary Table S12). Stearoyl-CoA desaturase is encoded by $S C D$ and catalyzes the biosynthesis of monounsaturated fatty acids. Notably, stearoyl-CoA desaturase has been implicated in insulin resistance (IR) together with $T C F 7 L 2^{50,51}$. Collectively these results support the hypothesis that the TCF7L2 locus acts as a regulatory hub for genes involved in immune regulation as well as genes important in the etiology of T2D.

Cross-tissue eQTL enrichment of associations in T1D and T2D. We mapped tissue-specific regulatory networks, leveraging information on eQTL effects from the CoDeS3D analysis (Supplementary Table S2; Supplementary Table S3), to identify the tissues in which the disease-associated loci are most likely functional (i.e. eQTL-eGene-tissue triads). Consistent with previous observations ${ }^{22,23}$, we found that eQTL effects for T1D and T2D were variably distributed across different tissues (Fig. 4). The top-ranked tissues with the highest number of functional eQTL-eGene interactions for T1D SNPs included whole blood, thyroid, skin, and adipose subcutaneous tissues (Fig. 4). Thyroid, tibial nerve, skin and adipose subcutaneous tissues had the greatest numbers of regulatory impacts involving T2D eQTLs (Fig. 4). Tissue-specific enrichment analysis using TissueEnrich (R package) identified thyroid tissue as having the highest level of enrichment for expression of the genes that were regulated by eQTLs associated with T2D, while lymph nodes, lung and spleen were the most enriched tissues for genes regulated by T1D eQTLs (Supplementary Fig. S3).

It has been recently demonstrated that eQTLs associated with complex traits can have opposing effects on gene regulation in different tissues ${ }^{52}$. Therefore, we sought to determine whether T1D and T2D eQTL effects on the 165 pleiotropic genes (excluding 30 HLA genes) occurred in the same or opposite directions. We observed that a number of eQTLs impacted on the expression of shared genes in opposing directions across the same tissues (Supplementary Table S13). For example, T1D cis-eQTLs rs12598357, rs12928404, and rs4788084 downregulated SULT1A1 transcript levels in the pancreas. By contrast, a T2D cis-eQTL rs8046545 upregulated SULT1A1 
a)

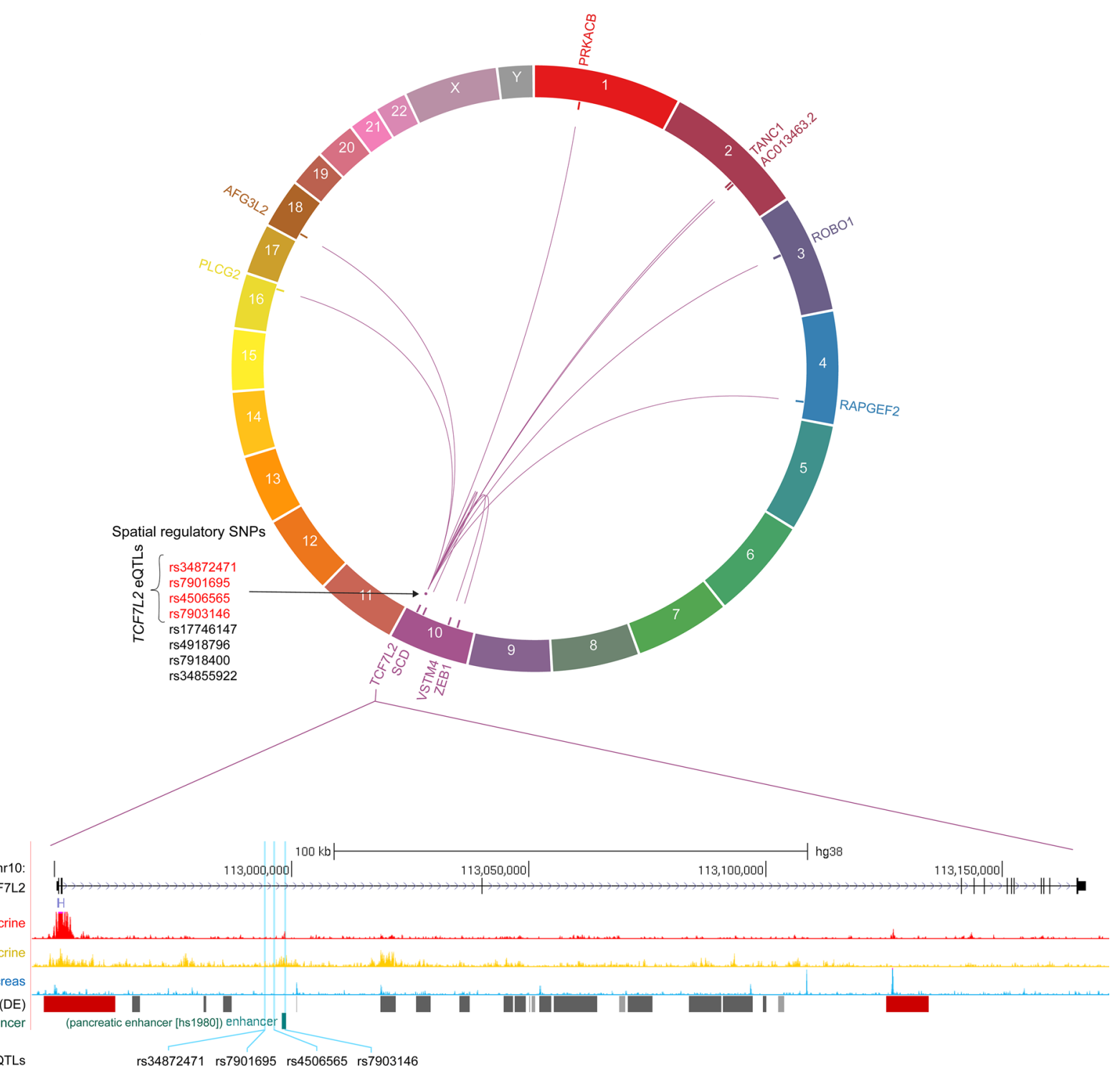

b)

Figure 3. Regulatory SNPs within the TCF7L2 locus modulate the expression of genes involved in immune regulation and genes important for the etiology of T2D. (a) A circos plot of significant regulatory interactions (i.e. innermost link lines) between SNPs within the TCF7L2 locus (i.e. the pointed purple dot) and the spatially regulated genes (i.e. labelled genes in the outermost track) at FDR $<0.05$. Spatial regulatory SNPs modulating the expression of TCF7L2 are highlighted. Linkage disequilibrium between genetic variants was obtained from https://ldlink.nci.nih.gov/. (b) An expanded view of the TCF7L2 gene locus. SNP rs7903146 overlaps histone modification marks (i.e. pancreatic $\mathrm{H} 3 \mathrm{~K} 4 \mathrm{me} 3$ and $\mathrm{H} 3 \mathrm{~K} 27 \mathrm{ac}$ ) and an annotated enhancer in the pancreas. Genome regulatory tracks were obtained from UCSC browser using hg38 coordinates chr10:112945186113172435 (https://genome.ucsc.edu). GH Reg Elems (DE)-GeneHancer regulatory elements (double elite). Circa software was used to generate the circos plot and is available at http://omgenomics.com/circa/. The annotated pancreatic enhancer (hs1980) was extracted from VISTA enhancer database ${ }^{94}$.

in the pancreas (Fig. 5a). Similarly, SULT1A2 pancreatic transcript levels were upregulated by T1D cis-eQTLs (i.e. rs12598357, rs12928404, rs4788084). Again, the T2D-associated cis-eQTL (i.e. rs8046545) downregulates SULT1A2 pancreatic transcript levels (Fig. 5a). Of the SULT1A2 transcript levels regulating eQTLs, only rs8046545 and rs12928404 are in strong LD $\left(\mathrm{R}^{2}>0.78\right)$ in people of European ancestry (Fig. 5a).

We also identified instances where T1D and T2D eQTLs modulate the transcript levels of shared genes in the same direction. For example, both rs151234 (T1D eQTL) and rs8046545 (T2D eQTL) downregulated the expression of SULT1A2 and TUFM in adipose and pancreas, respectively (Fig. 5b). In addition, both rs3130501 and rs3132524 (T2D eQTLs), together with rs3129889 (T1D eQTL), co-modulate the expression of AGPAT1 in the same direction in the liver (Fig. 5b). Notably, rs151234 is not in linkage with $\mathrm{rs} 8046545\left(\mathrm{R}^{2}<0.1\right)$, consistent with the SNPs marking distinct spatial regulatory elements that are not co-inherited.

We observed that trans-eQTLs have mixed effects on transcript levels of shared genes. For example, rs12203596 (T1D eQTL) upregulated IGF2BP2 in the transverse colon, while rs35261542 (T2D eQTL) 


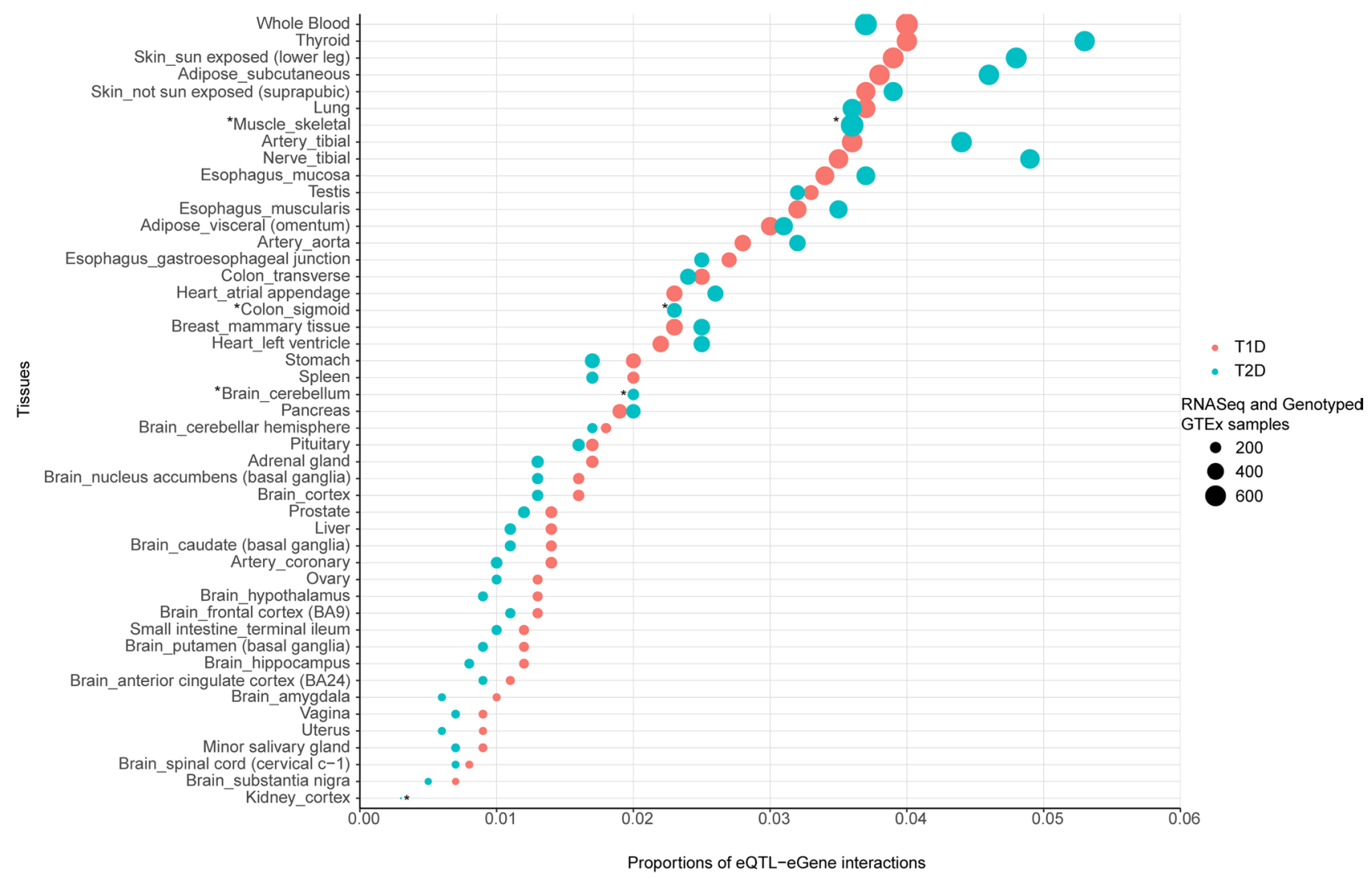

Figure 4. Functional eQTL effects are variably distributed across tissues. The proportions of significant T1D and T2D eQTL-eGene interactions across tissues (x-axis) in comparison to number of GTEx tissue samples (circles). The top ranked tissues with the highest number of functional T1D eQTL-eGene interactions include whole blood, thyroid, skin and adipose tissues. T2D eQTLs were greatest in the thyroid, tibial nerve, skin and adipose subcutaneous tissues. ${ }^{*}$ Tissues with the same number of functional T1D and T2D eQTL-eGenes interactions.

downregulated IGF2BP2 expression in the terminal ileum tissue (Fig. 5c). By contrast, APOM expression was downregulated by rs9273368 (trans-eQTL associated with T1D, T2D, and LADA) and rs9273369 (trans-eQTL associated only with T1D) in skeletal muscle tissues (Fig. 5c). Notably, rs9273368 and rs9273369 are strongly co-inherited $\left(\mathrm{R}^{2}>0.8\right)$ in the African Yoruba population but not people of other ancestries.

Collectively, these results indicate that: (a) eQTL effects for T1D and T2D SNPs have tissue-specific effects on gene expression; and (b) T1D and T2D SNPs can co-regulate genes in the same tissue consistent with the existence of converging biological pathways.

Protein-protein interaction network identifies drug repurposing targets. Traits that share core genes or whose genes interact closely in biological pathways are hypothesized to have correlated effects ${ }^{25}$. Therefore, we used STRING (Methods; http://string-db.org; version $11^{53}$ ) to construct the protein-protein interaction (PPI) network for the 165 shared genes associated with both T1D and T2D. Of the 165 genes analyzed, we identified 137 nodes (i.e. functional proteins encoded by the genes) and 117 edges (i.e. predicted functional associations) at a significant PPI enrichment of $p<1.0 \times 10^{-16}$ (Fig. 6; Supplementary Table S14). STRING identified 6 PPI clusters (circled) using the K-means clustering algorithm $(\mathrm{K}-\mathrm{Means}=6)$ of functional biological interactions within the overall network (Methods; Fig. 6; Supplementary Table S15). These clusters included, for example, the hub of highly connected genes (i.e. METTL15, SAMM50, PMPCA, SH2B1 and ATG16L1; Fig. 6; Supplementary Table S14) about the TUFM gene, which encodes the mitochondrial translation elongation factor. This TUFMassociated hub is enriched in regulatory proteins important for mitochondrial function ${ }^{54-57}$, consistent with the central role that mitochondrial dysfunction is hypothesised to have in diabetes ${ }^{58,59}$.

We hypothesized that the proteins within our PPI network represented high value targets for therapeutic development. Therefore, we mined the Drug Gene Interaction database (DGIdb) to identify shared genes that encode proteins that are affected by at least one drug within the interaction networks. We identified that 25 of the 165 genes $(\sim 15 \%)$ encode proteins affected by FDA approved drugs (Supplementary Table S16). The proteins encoded by these genes interact directly with other proteins within the PPI network (Fig. 6). Some of the drugs we identified (e.g. streptozotocin, pembrolizumab, nivolumab, and doxorubicin) cause diabetes or diabetes-like symptoms (i.e. hyperglycemia) as side-effects of usage. For example, streptozotocin affects the proteins encoded by TH and SULT1A2 (Supplementary Table S16), and has been widely used experimentally to induce diabetes 
a)

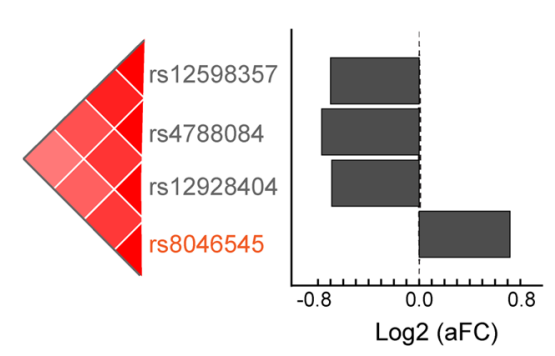

b)

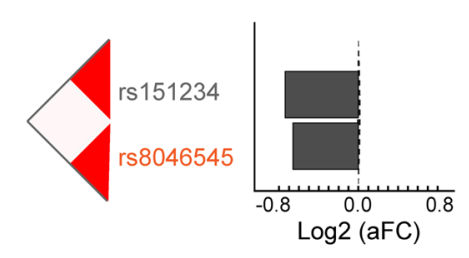

c)

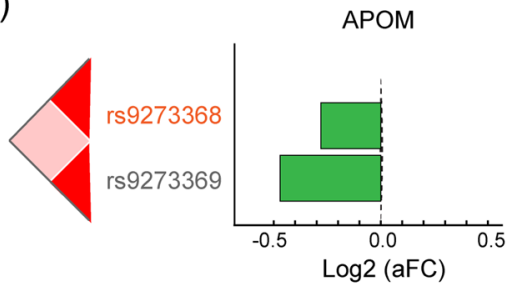

SULT1A2

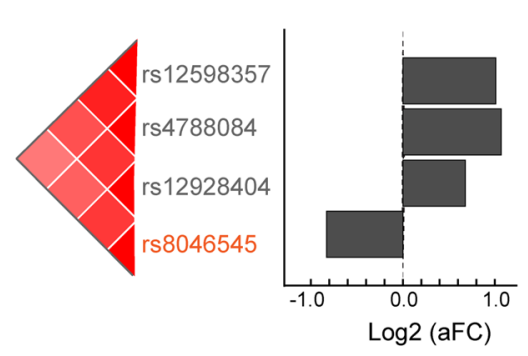

SULT1A2

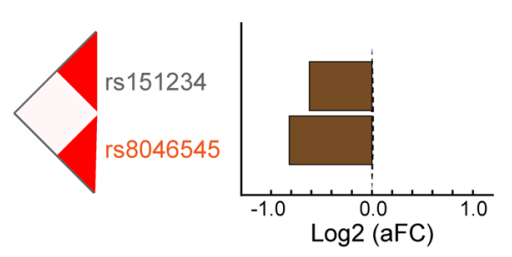

IGF2BP2

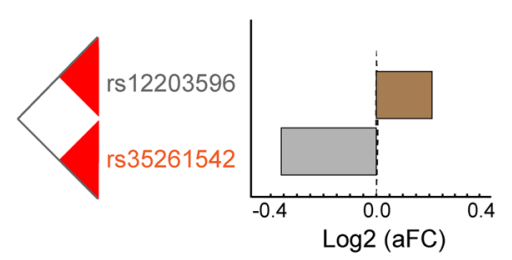

SH2B1
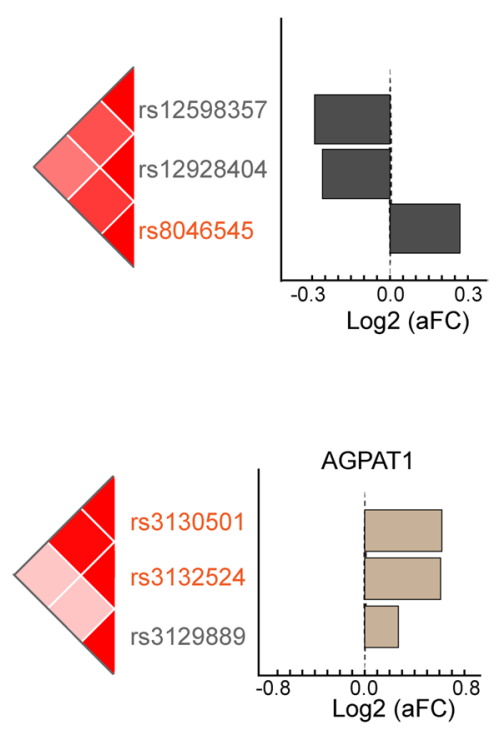

DGKB

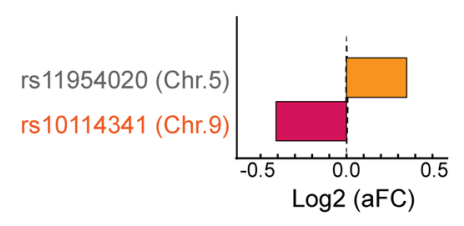

KEY:

rsID T1D

rsID T2D

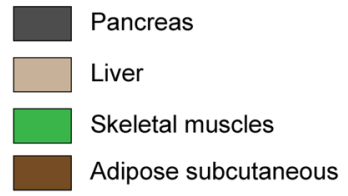

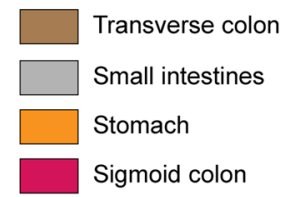

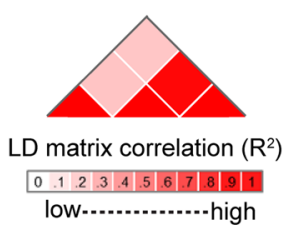

Figure 5. T1D and T2D eQTLs have tissue-dependent effects across human tissues. (a) T1D and T2D ciseQTLs are associated with spatial regulation of shared genes in the pancreas but in opposite directions. (b) T1D and T2D cis-eQTLs have similar eQTL effects on shared genes across tissues. (c) Trans-eQTLs have mixed effects on transcript levels of shared genes across tissues. aFC-allelic fold change, which denotes the direction of eQTL effect [i.e. upregulated $(+)$ or downregulated (-)]. cis-interactions within $1 \mathrm{Mb}$ on the same chromosome; trans-interactions $>1 \mathrm{Mb}$ either on the same chromosome or interactions $>1 \mathrm{Mb}$ on different chromosomes. Linkage matrices between SNPs are based on European population and were obtained from https://ldink.nci.nih.gov/.

in rodent models due to its toxic effects on pancreatic beta cells ${ }^{60}$. Pembrolizumab and nivolumab (targeting the protein encoded by MSH3) have been reported to induce the development of insulin-dependent diabetes in cancer patients ${ }^{61}$. Similarly, doxorubicin (targeting the protein encoded by ZEB1) has been shown to result in severe hyperglycemia and IR in an experimental rat model ${ }^{62}$ (Supplementary Table S16).

Not all of the side-effects are negative. For example, sirolimus, affects the MAPK10 gene product, has been shown to normalize glucose metabolism in diabetic mice ${ }^{63,64}$, decrease IR in diabetic rats ${ }^{65}$, and prevent IR in humans $^{66}$ (Supplementary Table S16). Similarly, mitomycin (another compound that affects the MAPK10 gene product) has been hypothesized to suppress pro-inflammatory events and cause the induction of regulatory $\mathrm{T}$ cells differentiation following islet allograft transplantation ${ }^{67}$.

Several of the drugs we identified have been trialed or repurposed for the treatment of diabetes. For example, disulfiram (targeting $A L D H 2$ gene product) has recently been shown to normalize body weight and restore insulin responsiveness in obese mice ${ }^{68}$. Similarly, pirfenidone and tretinoin (Supplementary Table S16) have been trialed for the management of long-term diabetic complications, i.e. diabetic nephropathy and diabetic footulcers, respectivel ${ }^{69,70}$. Collectively, our results highlight the potential for a genetics-informed and network-based 


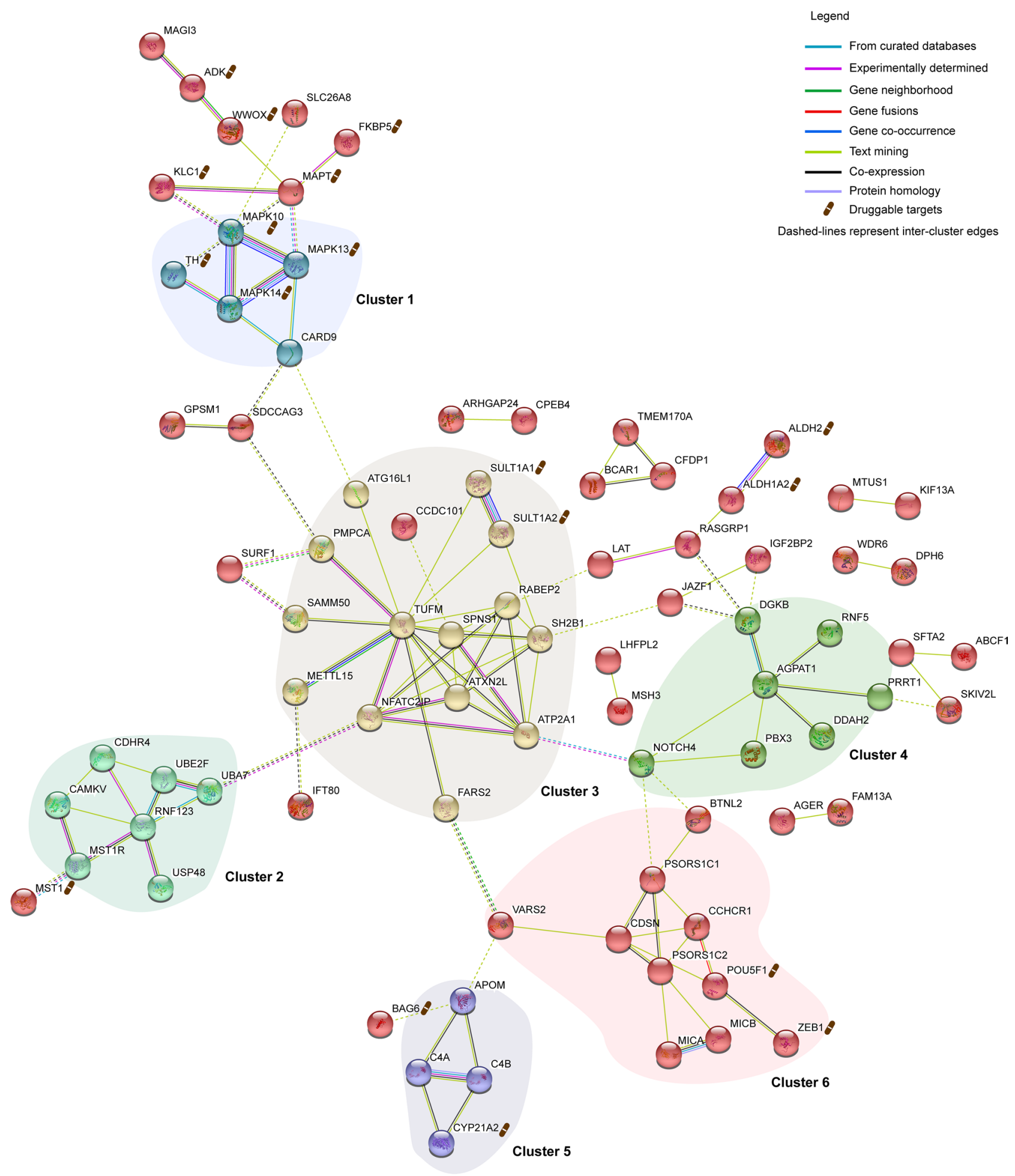

Figure 6. Protein-protein interaction network identifies existing drug targets that may impact diabetes. A protein-protein interaction network for the pleiotropic genes was constructed using string-db-org (http:// string-db.org; version $11^{53}$ ) set to a medium confidence of 0.400 and prediction methods (i.e. genomic context prediction channels-neighbourhood, fusion, and co-occurrence; together with co-expression, text mining, curated databases, and experiments). 137 nodes (i.e. genes encoding functional proteins) were identified from an input of 165 genes at a PPI enrichment of $p<1.0 \times 10^{-16}$. Nodes with at least 2 interactions are shown. PPI clusters were identified using K-means clustering algorithm set to 6 clusters $(\mathrm{K}$-means $=6)$. Dashed lines represent inter-cluster edges. Proteins with a drug symbols are targets for existing FDA-approved drugs from the Drug Gene Interaction database (DGIdb; v3.0.2). A summary of the protein interaction network with the respective interaction scores is available in Supplementary Table S14. A summary of the drug-gene interactions from DGIdb is available in Supplementary Table S16. 
approach to understand and prevent adverse reactions while providing an avenue for repurposing existing drugs in the management of diabetes.

\section{Discussion}

We have identified how genetic variation associated with T1D and T2D impacts on common biological pathways through putative gene regulatory networks that include both shared and unique genes. Our data show that spatial eQTLs nearby T1D and T2D associated genetic variants share downstream pathways. Notably, transcript levels of genes associated with eQTLs for the highly discriminatory PRS for T1D (T1D-GRS2) showed greater overlap with T2D-associated eQTLs than was observed when comparing transcript levels of genes associated with T2D-GRS and T1D-associated eQTLs. Yet, there was no significant genetic correlation detected in people of European ancestry (using UKBB data). Thus, our findings are consistent with forms of T1D and T2D having overlapping biological mechanisms that arise from regulatory impacts on shared genes and pathways. However, it appears that a genetic risk profile for T1D alters more biological pathways that increase the risk of developing both T1D and T2D, than the reverse.

It has been suggested that the development of complex 'related' traits can be driven by tissue-and diseasespecific eQTL effects on the regulation of common genes ${ }^{52}$. Consistent with this, we observed upregulation of $S H 2 B 1$ in adipose and pancreatic tissues by a T2D eQTL, and downregulation of $S H 2 B 1$ expression by a T1D eQTL across the same tissues. While the eQTLs we identified are associated with a life-long reduction or increase in expression relative to the reference genotype, this is still environmentally modifiable by epigenetic mechanisms ${ }^{52}$. However, it is worth noting that hypothalamic overexpression of $S H 2 B 1$ was recently reported in a mouse model to protect against obesity and metabolic disease, including diet-induced $\mathrm{IR}^{71}$.

Further support for the impact of disease-specific genetic variation on shared genes is obtained from the opposing T1D and T2D trans-eQTL effects on diacylglycerol kinase beta (encoded by DGKB), whose kinase family has been implicated in peripheral IR and abnormal glucose uptake ${ }^{72}$. Similarly, T1D- and T2D-specific eQTL effects were observed on SULT1A1 and SULT1A2, which encode enzymes involved in amine metabolism and lipid metabolic pathways ${ }^{73}$. Therefore, we contend that our results support the hypothesis that genetic risk impact tissue-specific regulation of shared genes, thereby influencing the etiology of T1D and T2D through similar metabolic pathways but different mechanisms.

The protein-protein interaction network we identified revealed an intricate metabolic network for the shared genes regulated by both T1D and T2D eQTLs. For example, a hub protein, apolipoprotein M (encoded by $A P O M$ ), is a key regulator of high-density lipoprotein metabolism that subsequently modulates the efflux of cholesterol and atherosclerosis susceptibility ${ }^{74}$. Another hub protein, AGPAT1, together with AGPAT2, has important roles in the biosynthesis of glycerophospholipids and is hypothesized to play a role in the development of $\mathrm{IR}^{75}$. Notably, IR is a prominent feature for both T1D and T2D and has been demonstrated to impact on lipid and lipoprotein metabolism, ultimately resulting in dyslipidemia and diabetes-associated vascular complications ${ }^{76-78}$.

Diabetes is a very heterogeneous disease in regard to the clinical, genetic, immunologic, and metabolic features that define disease onset and progression. Notably, genetic risk scores for T1D and T2D have been instrumental in predicting disease-onset ${ }^{40,41}$. However, transcriptional risk scores (TRS) have been reported to outperform genetic risk scores in distinguishing patients with Crohn disease from healthy subjects and predicting disease progression ${ }^{79}$. Therefore, since most disease-associated SNPs regulate transcript levels of genes (which is in a sense closer to the phenotype), understanding how these SNPs influence gene expression is important to identify genes whose association with disease is either through protection, promotion, or pleiotropy ${ }^{79}$. For instance, the autoimmune LADA phenotype is considered a genetic admixture of T1D and T2D due to its association with TCF7L2, a transcription factor that is also associated with T2D risk ${ }^{16}$.

Interestingly, our analysis identified spatial regulatory elements within the TCF7L2 locus associated with the expression of immune regulatory genes, as well as genes involved in insulin signaling pathways. One key finding was the identification that T2D eQTLs, within TCF7L2, and a T1D eQTL trans-regulate ZEB1 gene. ZEB1 encodes a zinc finger transcription factor that functions as a key regulator of the $\mathrm{T}$ cell signaling and differentiation in the thymus ${ }^{49}$. Therefore, we contend that the TCF7L2 locus encompasses a regulatory hub for genes important for the etiology of T1D and T2D. Our conclusion corroborates observations of TCF7L2 associated gene regulatory impacts $^{80}$ and studies reporting that TCF7L2 SNPs are associated with the presence of islet autoantibodies in $\mathrm{LADA}^{17}$, and autoantibody positivity in recent-onset T1D patients ${ }^{45}$.

Our study identified targets for drugs associated with adverse reactions through the integration of PPI networks and drug-gene interactions. For example, streptozotocin, which targets pleiotropic proteins, has been demonstrated to induce diabetes in rodent models ${ }^{60}$. By contrast, sirolimus is reported to prevent IR in humans ${ }^{66}$. At the same time, the efficacy of pirfenidone and tretinoin has been evaluated in the management of diabetic nephropathy and foot-ulcers, respectively ${ }^{69,70}$. Moreover, disulfiram, which is used for the treatment of alcoholism, has been shown to normalize fat mass and insulin sensitivity in diet-induced obese mice and repurposing of this drug in the clinic has been suggested as a strategy to treat obesity and related metabolic complications ${ }^{68}$. Notably, studies on monogenic forms of diabetes such as neonatal diabetes have provided a proof-of-concept that an individual's genotype can guide on the treatment modality ${ }^{81}$. Therefore, it seems plausible that geneticsinformed and network-based prescription could provide an avenue for repurposing existing drugs while preventing adverse drug reactions.

Our study has limitations. Firstly, our genetic correlation and colocalization analyses were performed using genome-wide genotype data of individuals of the European ancestry, reflecting that over $90 \%$ of GWA studies on T1D have been performed in populations of European ancestry. Secondly, the colocalization test assumes a single causal variant for a trait ${ }^{28}$. Moreover, it ignores the fact that transcript levels of genes can be modified through various mechanisms, not all of which are necessarily associated with disease risk ${ }^{28}$. Furthermore, the 
lack of complete colocalization between disease and eQTL signals could indicate that the right SNP-gene-tissue triads were eliminated from the tests by selecting only the SNP-gene pairs with the lowest $p$ values. Nonetheless, our analyses revealed partial colocalization between disease and spatial eQTL signals. Therefore, we contend that experimental manipulation through CRISPR will be required to establish causality. Thirdly, the genetic admixture of GWAS SNPs, together with the inclusion of GWAS SNPs associated with phenotypes that are not classically defined as T1D could limit the generalization of pleiotropic effects. Nonetheless, the inclusion of highly predictive SNPs used in polygenic risk scores for T1D and T2D from populations with fairly similar genetic linkage strongly supports the identified co-transcriptional regulation of shared genes for T1D and T2D. Fourthly, our analysis involved the use of datasets from 'whole' pancreatic tissue, which contains a mixture of endocrine and exocrine cells. Future studies should limit their analyses to single cell types to confirm the pleiotropy we identified. Finally, the integration of extensive $\mathrm{Hi}-\mathrm{C}$ datasets increases the power to detect more cell type and developmental stage-specific functional chromatin interactions to understand the genetic basis of complex diseases at the systems-level. However, as the number of tests increases, correcting for multiple testing using the Benjamini-Hochberg $(\mathrm{BH})$ procedure is conservative ${ }^{82}$. This could potentially result in an under-estimate of the extent of shared gene overlap between T1D and T2D, thereby underestimating the identification of pleiotropic genes. Nevertheless, the $\mathrm{BH}$ procedure corrects for multiple testing by ranking $p$ values ${ }^{82}$, which ensures a very high probability of true-positives, thereby increasing the confidence of eQTL associations.

\section{Conclusion}

Our findings support the existence of common genetic regulatory mechanisms that co-regulate genes that mediate pleiotropic effects on T1D and T2D. Importantly, our results further support the role of TCF7L2 locus, a well-known T2D susceptibility region, as a key regulatory hub that modulates transcript levels of genes involved in immune regulation as well as genes important in the etiology of T2D. Empirical studies that integrate genome editing techniques (i.e. CRISPR-Cas9) will further refine our understanding of these regulatory interactions and their roles in the development of islet autoimmunity, T1D and T2D.

\section{Methods}

Identification of SNPs associated with the development of T1D and T2D. The genetic variants used in this study were genotyped and imputed SNPs associated with T1D obtained from: the GWAS catalog (a keyword search for "Type 1 diabetes" was performed and associations were selected based on a $p$ value threshold $\left[p\right.$ values $\left.<5 \times 10^{-6}\right]$; http://www.ebi.ac.uk/gwas; v1.0.1; downloaded March 25, 2020) (Supplementary Table S4); studies on polygenic risk scores for T1D ${ }^{40,83,84}$; prospective studies ${ }^{10,85-87}$; and time-to-event studies ${ }^{88,89}$. For the T2D-associated genetic variants, SNPs were obtained from the GWAS catalog (a keyword search for "Type 2 diabetes" was performed and associations were selected based on a $p$ value threshold [ $p$ values $\left.<5 \times 10^{-6}\right]$; http:// www.ebi.ac.uk/gwas; v1.0.1; downloaded April 8, 2020) (Supplementary Table S4), and a study on T2D polygenic risk scores ${ }^{41}$. A total of 346 T1D SNPs and 1,569 T2D SNPs were used in the eQTL analysis (Supplementary Table S5). Genomic positions for SNPs are annotated according to reference human hg38 genome build.

Identification of spatial eQTL-eGene pairs for T1D and T2D-associated SNPs. We used the Contextualizing Developmental SNPs in 3-Dimensions (CoDeS3D) algorithm as described in ${ }^{26}$ to identify SNPs associated with the spatial regulation of gene transcript levels through physical interactions. Briefly, the CoDeS3D modular python scripts integrate Hi-C contact libraries from published sources (Supplementary Table S1) to identify spatial co-localization of two DNA fragments, with one fragment marking the queried SNP. Gene-containing restricted fragments that are in physical contact with fragments containing the queried SNPs are identified as spatial pairs to the SNPs. Finally, the resultant spatial SNP-gene pairs are queried in the Genotype-Tissue Expression database (GTEx) to identify SNPs that are associated with transcript levels of genes through physical interaction at $\mathrm{FDR}<0.05^{26}$.

Here, we integrated extensive $\mathrm{Hi}-\mathrm{C}$ contact libraries to identify all possible tissue, cell type and developmental stage-specific chromatin interactions based on the emerging evidence that complex diseases culminate from systems-level perturbations ${ }^{24,25}$. First, the spatial interactions were identified from Hi-C contact libraries captured from: (1) primary human tissues (i.e. including pancreas, liver, lung, spleen, muscle, and adrenal gland); (2) primary and immortalized immune cell-types (i.e. B and T lymphocytes); and (3) embryonic stem cells, including cell lines representing embryonic germ layers (Supplementary Table S1). Next, the regulatory potential of the identified SNP-gene pairs was tested through the integration of expression QTL information from 47 human tissues and 2 immortalized cell-lines (Genotype-Tissue Expression database [GTEx] v8; http:// www.gtexportal.org ${ }^{90}$ ).

Spatial eQTLs were deemed significant and recorded if the FDR $<0.05$ after correcting for multiple testing using the $\mathrm{BH}$ procedure ${ }^{82}$. Finally, genes whose transcript levels were associated with a spatial-eQTL were denoted as eGenes. The eQTL-eGene interactions were defined as either cis (i.e. interactions within $1 \mathrm{Mb}$ on the same chromosome), trans-intrachromosomal (i.e. interactions $>1 \mathrm{Mb}$ but on the same chromosome), or transinterchromosomal (i.e. interactions $>1 \mathrm{Mb}$ but on the different chromosomes). All datasets and analyses were prepared and carried out using the human genome reference build GRCh38.p7. Genomic positions for eGenes derived from GTEx are annotated according to GENCODE v25. The HLA genes were excluded from the shared genes analyses because we wanted to identify HLA independent key pathways and networks since HLA genes are strongly associated with $\mathrm{T}_{1} \mathrm{D}^{12}$.

Genetic correlation and SNP heritability analyses. We employed the recently developed HDL method $^{42}$ to estimate the genetic correlation between T1D and T2D, together with obesity and body mass index 
(BMI) using population-level data from the UKBB. The genome-wide genotype data available in the UKBB is obtained from a large prospective cohort study of $\sim 500,000$ individuals across the United Kingdom, providing a rich resource for genetic analyses. Genetic correlation and SNP heritability analyses for the phenotypes in this report were conducted as described on https://github.com/zhenin/HDL/wiki. Briefly, the UKBB summary statistics of genome-wide associations for T1D, T2D, BMI and obesity were obtained from the Neale lab (i.e. round 2 association tests released in 2018; https://www.nealelab.is/uk-biobank/). The association tests on curated phenotypes were performed on 361,194 unrelated individuals of British ancestry as described on https://www.neale lab.is/uk-biobank/. Computed linkage disequilibrium matrices and imputed reference panels of HapMap3 SNPs (i.e. 1,029,876 quality-controlled UKBB imputed SNPs) were downloaded from https://github.com/zhenin/ HDL/wiki/Reference-panels. The imputed panel of SNPs was used as it provides a more accurate estimate of genetic correlations ${ }^{42}$.

Genetic colocalization analyses. Genetic colocalization analysis permits the identification of shared 'causal' SNPs or genes within a genomic loci across disease vs trait or trait vs trait association signals ${ }^{28}$. Bayesian colocalization tests between spatial cis-eQTL and disease-associated signals were performed for 7 genomic regions strongly associated $\left(p<10^{-8}\right)$ with both T1D and T2D (i.e. SH2B3, MAPK14, CTRB1/2, INS, ASCL2/ MIR4686, and HLA region) using the COLOC R package. Briefly, T1D and T2D GWAS summary statistics of individuals of European ancestry were accessed from https://gwas.mrcieu.ac.uk/ using R software package (gwasglue; https://github.com/mrcieu/gwasglue/), and SNPs extracted within $200 \mathrm{~kb}$ from the lead SNP. Spatial cis-eQTL summary data was derived from CoDeS3D analysis as described in Sect. 5.2. For each SNP, we selected SNP-gene pairs with the lowest $p$ value and performed colocalization (i.e. coloc.abf) test between disease and eQTL summary data with priors set as $p 1=1 \times 10^{-4}, p 2=1 \times 10^{-4}$, and $p 12=5 \times 10^{-5}$, as previously suggested ${ }^{28}$. In total, 2129 pairwise comparisons were examined for evidence of colocalization between eQTL and disease signals.

Pathway analysis and functional gene annotations. Biological pathway enrichments for the differentially expressed genes were identified using the R software package (g:Profiler ${ }^{27}$ ) with a significance threshold of $p$ value $<0.05$ threshold. R software package (TissueEnrich ${ }^{91}$ ) was used for the tissue-specific gene expression analysis. PubTator Central ${ }^{92}$ was used for manual literature curation to examine the molecular and phenotypic implications of specific examples of differentially expressed genes.

The construction of the PPI network for the pleiotropic genes. We used the Retrieval of Interacting Genes/Proteins database (STRING; v.11 9.0) 53 $^{5}$ to construct a protein-protein interaction network for the differentially expressed genes associated with both T1D and T2D. The PPI network was set to a medium confidence of 0.400 with the following prediction evidence: (1) genomic context prediction channels-neighborhood, fusion, and co-occurrence; (2) co-expression; (3) text mining; (4) curated databases; and (4) experiments. PPI clusters were identified using K-means clustering algorithm. The drug-gene interaction database (DGIdb; v3.0.2 $)^{93}$ was mined to identify genes that encode proteins that are targets for at least a single FDA-approved drug within the PPI network.

Data analysis. Statistical testing, visualization, and genetic correlations analyses were performed using $\mathrm{R}$ software (v3.6.3) and RStudio (version 1.2.5042-1). Python version 3.7.6 was used for the bootstrap analysis. Scripts for genetic colocalization, data analysis and visualization can be accessed on Figshare with the identifier https://doi.org/10.17608/k6.auckland.12886745.

Code and data accessibility. The CoDeS3D pipeline is available at: https://github.com/Genome3d/codes 3d-v2/. HDL software is available at https://github.com/zhenin/HDL/. GWAS catalog can be accessed at http:// www.ebi.ac.uk/gwas/. UKBB summary statistics from the Neale lab are available at https://www.nealelab.is/ukbiobank/. GTEx portal can be accessed at http://www.gtexportal.org/. The UCSC browser is accessed at https:// genome.ucsc.edu/. The linkage disequilibrium matrix (LDlink) is available from https://ldink.nci.nih.gov/. R software package, gwasglue, is accessed at https://github.com/MRCIEU/gwasglue. Circa software for generating circos plots is available at http://omgenomics.com/circa/. The STRING database can be accessed at http://stringdb.org/. The Drug Gene Interaction database (DGIdb) can be accessed at http://www.dgidb.org/.

\section{Data availability}

All data generated or analyzed during this study are included in this published article (and its Supplementary Information files [Supplementary Tables S1-S16]).

Received: 7 October 2020; Accepted: 16 June 2021

Published online: 06 July 2021

\section{References}

1. Mathis, D., Vence, L. \& Benoist, C. $\beta$-Cell death during progression to diabetes. Nature 414, 792-798 (2001).

2. Wilkin, T. J. The accelerator hypothesis: weight gain as the missing link between type I and type II diabetes. Diabetologia 44, 914-922 (2001).

3. Donath, M. Y., Størling, J., Maedler, K. \& Mandrup-Poulsen, T. Inflammatory mediators and islet $\beta$-cell failure: a link between type 1 and type 2 diabetes. J. Mol. Med. 81, 455-470 (2003). 
4. Hamman, R. F. et al. The SEARCH for Diabetes in Youth Study: rationale, findings, and future directions. Diabetes Care 37, 3336-3344 (2014).

5. Tuomi, T. et al. Clinical and genetic characteristics of type 2 diabetes with and without GAD antibodies. Diabetes 48, 150-157 (1999).

6. Tuomi, T. et al. Antibodies to glutamic acid decarboxylase reveal latent autoimmune diabetes mellitus in adults with a non-insulindependent onset of disease. Diabetes 42, 359-362 (1993).

7. Eftychi, C. et al. Analysis of the type 2 diabetes-associated single nucleotide polymorphisms in the genes IRS1, KCNJ11, and PPARG2 in type 1 diabetes. Diabetes 53, 870-873 (2004).

8. Xue, A. et al. Genome-wide association analyses identify 143 risk variants and putative regulatory mechanisms for type 2 diabetes. Nat. Commun. 9, 2941 (2018).

9. Vujkovic, M. et al. Discovery of 318 new risk loci for type 2 diabetes and related vascular outcomes among 1.4 million participants in a multi-ancestry meta-analysis. Nat. Genet. 52, 680-691 (2020).

10. Sharma, A. et al. Identification of non-HLA genes associated with development of islet autoimmunity and type 1 diabetes in the prospective TEDDY cohort. J. Autoimmun. 89, 90-100 (2018).

11. Li, H. et al. Possible human leukocyte antigen-mediated genetic interaction between type 1 and type 2 diabetes. J. Clin. Endocrinol. Metab. 86, 574-582 (2001).

12. Lambert, A. P. et al. Absolute risk of childhood-onset type 1 diabetes defined by human leukocyte antigen class II genotype: a population-based study in the United Kingdom. J. Clin. Endocrinol. Metab. 89, 4037-4043 (2004).

13. Tuomilehto-Wolf, E. et al. Genetic susceptibility to non-insulin dependent diabetes mellitus and glucose intolerance are located in HLA region. BMJ 307, 155-159 (1993).

14. Ma, Z.-J., Sun, P., Guo, G., Zhang, R. \& Chen, L.-M. Association of the HLA-DQA1 and HLA-DQB1 Alleles in type 2 diabetes mellitus and diabetic nephropathy in the Han ethnicity of China. J. Diabetes Res. 2013, 1-5 (2013).

15. Williams, R. C. et al. HLA-DRB1 reduces the risk of type 2 diabetes mellitus by increased insulin secretion. Diabetologia 54, 1684-1692 (2011)

16. Lukacs, K. et al. The type 2 diabetes-associated variant in TCF7L2 is associated with latent autoimmune diabetes in adult Europeans and the gene effect is modified by obesity: a meta-analysis and an individual study. Diabetologia 55, 689-693 (2012).

17. Zampetti, S. et al. Association of TCF7L2 gene variants with low GAD autoantibody titre in LADA subjects (NIRAD Study 5). Diabet. Med. 27, 701-704 (2010).

18. Cervin, C. et al. Genetic similarities between latent autoimmune diabetes in adults, type 1 diabetes, and type 2 diabetes. Diabetes 57, 1433-1437 (2008).

19. Aylward, A., Chiou, J., Okino, M.-L., Kadakia, N. \& Gaulton, K. J. Shared genetic risk contributes to type 1 and type 2 diabetes etiology. Hum. Mol. Genet. https://doi.org/10.1093/hmg/ddy314 (2018).

20. Grant, S. F. A. The TCF7L2 locus: a genetic window into the pathogenesis of type 1 and type 2 diabetes. Diabetes Care 42, 1624-1629 (2019).

21. American Diabetes Association. Diagnosis and classification of diabetes mellitus. Diabetes Care 36, S67-S74 (2013).

22. Fadason, T., Ekblad, C., Ingram, J. R., Schierding, W. S. \& O'Sullivan, J. M. Physical interactions and expression quantitative traits loci identify regulatory connections for obesity and type 2 diabetes associated SNPs. Front. Genet. 8, 1-12 (2017).

23. Nyaga, D. M., Vickers, M. H., Jefferies, C., Perry, J. K. \& O'Sullivan, J. M. Type 1 diabetes mellitus-associated genetic variants contribute to overlapping immune regulatory networks. Front. Genet. 9, 535 (2018).

24. Yang, X. Multitissue multiomics systems biology to dissect complex diseases. Trends Mol. Med. 26, 718-728 (2020).

25. Boyle, E. A., Li, Y. I. \& Pritchard, J. K. An expanded view of complex traits: from polygenic to omnigenic. Cell 169, 1177-1186 (2017).

26. Fadason, T., Schierding, W., Lumley, T. \& O’Sullivan, J. M. Chromatin interactions and expression quantitative trait loci reveal genetic drivers of multimorbidities. Nat. Commun. 9, 5198 (2018).

27. Raudvere, U. et al. g:Profiler: a web server for functional enrichment analysis and conversions of gene lists (2019 update). Nucleic Acids Res. 47, W191-W198 (2019).

28. Wallace, C. Eliciting priors and relaxing the single causal variant assumption in colocalisation analyses. PLOS Genet. 16, e1008720 (2020).

29. Nath, A. P. et al. Multivariate genome-wide association analysis of a cytokine network reveals variants with widespread immune, haematological, and cardiometabolic pleiotropy. Am. J. Hum. Genet. 105, 1076-1090 (2019).

30. Khoo, S. et al. MAP kinases and their roles in pancreatic $\beta$-cells. Cell Biochem. Biophys. 40, 191-200 (2004).

31. Kusari, A. B., Byon, J., Bandyopadhyay, D., Kenner, K. A. \& Kusari, J. Insulin-induced mitogen-activated protein (MAP) kinase phosphatase-1 (MKP-1) attenuates insulin-stimulated MAP kinase activity: a mechanism for the feedback inhibition of insulin signaling. Mol. Endocrinol. 11, 1532-1543 (1997).

32. Carlson, C. J., Koterski, S., Sciotti, R. J., Poccard, G. B. \& Rondinone, C. M. Enhanced basal activation of mitogen-activated protein kinases in adipocytes from type 2 diabetes: potential role of p38 in the downregulation of GLUT4 expression. Diabetes 52, 634-641 (2003).

33. Andersen, N. A., Larsen, C. M. \& Mandrup-Poulsen, T. TNF $\alpha$ and IFN $\gamma$ potentiate IL-1 $\beta$ induced mitogen activated protein kinase activity in rat pancreatic islets of Langerhans. Diabetologia 43, 1389-1396 (2000).

34. Furman, B. L., Wardlaw, A. C. \& Stevenson, L. Q. Bordetella pertussis-induced hyperinsulinaemia without marked hypoglycaemia: a paradox explained. Br. J. Exp. Pathol. 62, 504-511 (1981).

35. Yajima, M. et al. Islets-activating protein (IAP) in Bordetella pertussis that potentiates insulin secretory responses of rats. J. Biochem. 83, 295-303 (1978).

36. Gulbenkian, A., Schobert, L., Nixon, C. \& Tabachnick, I. I. A. Metabolic effects of pertussis sensitization in mice and rats. Endocrinology 83, 885-892 (1968).

37. Maries, E., Dass, B., Collier, T. J., Kordower, J. H. \& Steece-Collier, K. The role of a-synuclein in Parkinson's disease: insights from animal models. Nat. Rev. Neurosci. 4, 727-738 (2003).

38. Polymeropoulos, M. H. et al. Mutation in the alpha-synuclein gene identified in families with Parkinson's disease. Science 276, 2045-2047 (1997).

39. Geng, X. et al. a-Synuclein binds the K ATP channel at insulin-secretory granules and inhibits insulin secretion. Am. J. Physiol. Metab. 300, E276-E286 (2011).

40. Sharp, S. A. et al. Development and standardization of an improved type 1 diabetes genetic risk score for use in newborn screening and incident diagnosis. Diabetes Care 42, 200-207 (2019).

41. Vassy, J. L. et al. Polygenic type 2 diabetes prediction at the limit of common variant detection. Diabetes 63, 2172-2182 (2014).

42. Ning, Z., Pawitan, Y. \& Shen, X. High-definition likelihood inference of genetic correlations across human complex traits. Nat. Genet. https://doi.org/10.1038/s41588-020-0653-y (2020).

43. Carlsson, S., Ahlbom, A., Lichtenstein, P. \& Andersson, T. Shared genetic influence of BMI, physical activity and type 2 diabetes: a twin study. Diabetologia 56, 1031-1035 (2013).

44. Mayans, S. et al. TCF7L2 polymorphisms are associated with type 2 diabetes in northern Sweden. Eur. J. Hum. Genet. 15, 342-346 (2007). 
45. Redondo, M. J. et al. TCF7L2 genetic variants contribute to phenotypic heterogeneity of type 1 diabetes. Diabetes Care 41, 311-317 (2018).

46. Yu, P. et al. Autoimmunity and inflammation due to a gain-of-function mutation in phospholipase $\mathrm{C} \gamma 2$ that specifically increases external Ca2+ entry. Immunity 22, 451-465 (2005).

47. Fu, G., Chen, Y., Schuman, J., Wang, D. \& Wen, R. Phospholipase C $\gamma 2$ plays a role in TCR signal transduction and T cell selection. J. Immunol. 189, 2326-2332 (2012).

48. Glawe, J. D. et al. SDF-1-CXCR4 differentially regulates autoimmune diabetogenic T cell adhesion through ROBO1-SLIT2 interactions in mice. Diabetologia 56, 2222-2230 (2013).

49. Zhang, J. et al. Zeb1 represses TCR signaling, promotes the proliferation of T cell progenitors and is essential for NK1.1+ T cell development. Cell. Mol. Immunol. https://doi.org/10.1038/s41423-020-0459-y (2020).

50. Gutiérrez-Juárez, R. et al. Critical role of stearoyl-CoA desaturase-1 (SCD1) in the onset of diet-induced hepatic insulin resistance. J. Clin. Invest. 116, 1686-1695 (2006).

51. Oh, K.-J. et al. TCF7L2 modulates glucose homeostasis by regulating CREB- and FoxO1-dependent transcriptional pathway in the liver. PLoS Genet. 8, e1002986 (2012).

52. Mizuno, A. \& Okada, Y. Biological characterization of expression quantitative trait loci (eQTLs) showing tissue-specific opposite directional effects. Eur. J. Hum. Genet. 27, 1745-1756 (2019).

53. Szklarczyk, D. et al. STRING v11: protein-protein association networks with increased coverage, supporting functional discovery in genome-wide experimental datasets. Nucleic Acids Res. 47, D607-D613 (2019).

54. Lei, Y. et al. The mitochondrial proteins NLRX1 and TUFM form a complex that regulates type I interferon and autophagy. Immunity 36, 933-946 (2012).

55. Chen, H. et al. The human mitochondrial 12S rRNA m 4 C methyltransferase METTL15 is required for mitochondrial function. J. Biol. Chem. 295, 8505-8513 (2020).

56. Liu, S. et al. SAMM50 affects mitochondrial morphology through the association of Drp1 in mammalian cells. FEBS Lett. 590, 1313-1323 (2016).

57. Matsuzawa-Ishimoto, Y. et al. Autophagy protein ATG16L1 prevents necroptosis in the intestinal epithelium. J. Exp. Med. 214, 3687-3705 (2017).

58. Zabielski, P. et al. Altered skeletal muscle mitochondrial proteome as the basis of disruption of mitochondrial function in diabetic mice. Diabetes 65, 561-573 (2016).

59. Ruegsegger, G. N., Creo, A. L., Cortes, T. M., Dasari, S. \& Nair, K. S. Altered mitochondrial function in insulin-deficient and insulin-resistant states. J. Clin. Invest. 128, 3671-3681 (2018).

60. Furman, B. L. Streptozotocin-induced diabetic models in mice and rats. Curr. Protoc. Pharmacol. 70, 5.47.1-5.47.20 (2015).

61. Stamatouli, A. M. et al. Collateral damage: insulin-dependent diabetes induced with checkpoint inhibitors. Diabetes 67, 1471-1480 (2018).

62. de Lima Junior, E. A. et al. Doxorubicin caused severe hyperglycaemia and insulin resistance, mediated by inhibition in AMPk signalling in skeletal muscle. J. Cachexia. Sarcopenia Muscle 7, 615-625 (2016).

63. He, S. et al. Rapamycin/GABA combination treatment ameliorates diabetes in NOD mice. Mol. Immunol. 73, 130-137 (2016).

64. Reifsnyder, P. C., Flurkey, K., Te, A. \& Harrison, D. E. Rapamycin treatment benefits glucose metabolism in mouse models of type 2 diabetes. Aging (Albany, NY) 8, 3120-3130 (2016).

65. Zhou, W. \& Ye, S. Rapamycin improves insulin resistance and hepatic steatosis in type 2 diabetes rats through activation of autophagy. Cell Biol. Int. 42, 1282-1291 (2018).

66. Krebs, M. et al. The mammalian target of rapamycin pathway regulates nutrient-sensitive glucose uptake in man. Diabetes 56, 1600-1607 (2007).

67. Yamane, K. et al. Mitomycin C treatment improves pancreatic islet graft longevity in intraportal islet transplantation by suppressing proinflammatory response. Sci. Rep. 10, 12086 (2020).

68. Bernier, M. et al. Disulfiram treatment normalizes body weight in obese mice. Cell Metab. 32, 203-214.e4 (2020).

69. Sharma, K. et al. Pirfenidone for diabetic nephropathy. J. Am. Soc. Nephrol. 22, 1144-1151 (2011).

70. Tom, W. L., Peng, D. H., Allaei, A., Hsu, D. \& Hata, T. R. The effect of short-contact topical tretinoin therapy for foot ulcers in patients with diabetes. Arch. Dermatol. 141, 1373-1377 (2005).

71. Jiang, L. et al. Leptin receptor-expressing neuron Sh2b1 supports sympathetic nervous system and protects against obesity and metabolic disease. Nat. Commun. 11, 1517 (2020).

72. Chibalin, A. V. et al. Downregulation of diacylglycerol kinase delta contributes to hyperglycemia-induced insulin resistance. Cell 132, 375-386 (2008).

73. Chevereau, M., Glatt, H., Zalko, D., Cravedi, J.-P. \& Audebert, M. Role of human sulfotransferase $1 \mathrm{~A} 1$ and N-acetyltransferase 2 in the metabolic activation of 16 heterocyclic amines and related heterocyclics to genotoxicants in recombinant V79 cells. Arch. Toxicol. 91, 3175-3184 (2017).

74. Wolfrum, C., Poy, M. N. \& Stoffel, M. Apolipoprotein M is required for pre $\beta$-HDL formation and cholesterol efflux to HDL and protects against atherosclerosis. Nat. Med. 11, 418-422 (2005).

75. Agarwal, A. K. et al. Human 1-Acylglycerol-3-phosphate O -Acyltransferase Isoforms 1 and 2. J. Biol. Chem. 286, 37676-37691 (2011).

76. Guy, J. et al. Lipid and lipoprotein profiles in youth with and without type 1 diabetes: the SEARCH for Diabetes in Youth casecontrol Study. Diabetes Care 32, 416-420 (2009).

77. Xu, T. et al. Effects of metformin on metabolite profiles and LDL cholesterol in patients with type 2 diabetes. Diabetes Care 38, 1858-1867 (2015).

78. Cree-Green, M. et al. Delayed skeletal muscle mitochondrial ADP recovery in youth with type 1 diabetes relates to muscle insulin resistance. Diabetes 64, 383-392 (2015).

79. Marigorta, U. M. et al. Transcriptional risk scores link GWAS to eQTLs and predict complications in Crohn's disease. Nat. Genet. 49, 1517-1521 (2017).

80. Xia, Q. et al. The type 2 diabetes presumed causal variant within TCF7L2 resides in an element that controls the expression of ACSL5. Diabetologia 59, 2360-2368 (2016).

81. Bowman, P. et al. Effectiveness and safety of long-term treatment with sulfonylureas in patients with neonatal diabetes due to KCNJ11 mutations: an international cohort study. Lancet Diabetes Endocrinol. 6, 637-646 (2018).

82. Benjamini, Y. \& Hochberg, Y. Controlling the false discovery rate: a practical and powerful approach to multiple testing. J. R. Stat. Soc. B 57, 289-300 (1995).

83. Oram, R. A. et al. A type 1 diabetes genetic risk score can aid discrimination between type 1 and type 2 diabetes in young adults. Diabetes Care 39, 337-344 (2016).

84. Bonifacio, E. et al. Genetic scores to stratify risk of developing multiple islet autoantibodies and type 1 diabetes: a prospective study in children. PLOS Med. 15, e1002548 (2018).

85. Steck, A. K. et al. Improving prediction of type 1 diabetes by testing non-HLA genetic variants in addition to HLA markers. Pediatr. Diabetes 15, 355-362 (2014).

86. Frederiksen, B. N. et al. Evidence of stage- and age-related heterogeneity of non-HLA SNPs and risk of islet autoimmunity and type 1 diabetes: the Diabetes Autoimmunity Study in the Young. Clin. Dev. Immunol. 2013, 1-8 (2013). 
87. Bonifacio, E., Warncke, K., Winkler, C., Wallner, M. \& Ziegler, A.-G. Cesarean section and interferon-induced helicase gene polymorphisms combine to increase childhood type 1 diabetes risk. Diabetes 60, 3300-3306 (2011).

88. Steck, A. K. et al. Can non-HLA single nucleotide polymorphisms help stratify risk in TrialNet relatives at risk for type 1 diabetes?. J. Clin. Endocrinol. Metab. 102, 2873-2880 (2017).

89. Howson, J. M. M., Rosinger, S., Smyth, D. J., Boehm, B. O. \& Todd, J. A. Genetic analysis of adult-onset autoimmune diabetes. Diabetes 60, 2645-2653 (2011)

90. Aguet, F. et al. Genetic effects on gene expression across human tissues. Nature 550, 204-213 (2017).

91. Jain, A. \& Tuteja, G. TissueEnrich: Tissue-specific gene enrichment analysis. Bioinformatics 35, 1966-1967 (2019).

92. Wei, C.-H., Allot, A., Leaman, R. \& Lu, Z. PubTator central: automated concept annotation for biomedical full text articles. Nucleic Acids Res. 47, W587-W593 (2019).

93. Griffith, M. et al. DGIdb: mining the druggable genome. Nat. Methods 10, 1209-1210 (2013).

94. Visel, A., Minovitsky, S., Dubchak, I. \& Pennacchio, L. A. VISTA enhancer browser-a database of tissue-specific human enhancers. Nucleic Acids Res. 35, D88-D92 (2007).

\section{Acknowledgements}

The authors would like to thank the Genomics and Systems biology group for comments and discussion.

\section{Author contributions}

D.N. conducted analyses, interpreted data, and wrote the manuscript. M.V., C.J. and J.O. co-supervise D.N., participated in discussions and critically reviewed the final manuscript. T.F. updated CoDeS3D and commented on the manuscript. J.O. was responsible for overall direction of the study. All authors approved the final manuscript as submitted.

\section{Funding}

DMN was funded by the Sir Colin Giltrap Liggins Institute Scholarship. JOS was funded by grant UOAX1611: New Zealand-Australia Lifecourse Collaboration on Genes, Environment, Nutrition and Obesity (GENO) from the Ministry of Business, Innovation and Employment of New Zealand.

\section{Competing interests}

The authors declare no competing interests.

\section{Additional information}

Supplementary Information The online version contains supplementary material available at https://doi.org/ 10.1038/s41598-021-93346-x.

Correspondence and requests for materials should be addressed to J.M.O.

Reprints and permissions information is available at www.nature.com/reprints.

Publisher's note Springer Nature remains neutral with regard to jurisdictional claims in published maps and institutional affiliations.

(c) Open Access This article is licensed under a Creative Commons Attribution 4.0 International License, which permits use, sharing, adaptation, distribution and reproduction in any medium or format, as long as you give appropriate credit to the original author(s) and the source, provide a link to the Creative Commons licence, and indicate if changes were made. The images or other third party material in this article are included in the article's Creative Commons licence, unless indicated otherwise in a credit line to the material. If material is not included in the article's Creative Commons licence and your intended use is not permitted by statutory regulation or exceeds the permitted use, you will need to obtain permission directly from the copyright holder. To view a copy of this licence, visit http://creativecommons.org/licenses/by/4.0/.

(c) The Author(s) 2021 